\title{
An assessment of the solar irradiance record for climate studies
}

\author{
Greg Kopp* \\ University of Colorado, Laboratory for Atmospheric and Space Physics, 3665 Discovery Drive, Boulder, 80302 CO, USA \\ ${ }^{*}$ Corresponding author: Greg.Kopp@LASP.Colorado.edu
}

Received 17 September 2013 / Accepted 28 January 2014

\begin{abstract}
Total solar irradiance, the spatially and spectrally integrated radiant output from the Sun at a mean Sun-Earth distance of 1 astronomical unit, provides nearly all the energy driving the Earth's climate system. Variations in this energy, particularly over long time scales, contribute to changes in Earth's climate and have been linked to historical glaciation and inter-glacial periods as well as having a small effect on more recent global warming. Accurate measurements of solar irradiances require measurements above the Earth's atmosphere. The total solar irradiance spaceborne record began in 1978 and has been uninterrupted since, with over a dozen instruments contributing to the present solar climate data record. I assess the required and achieved accuracies of this record with a focus on its value for climate studies.
\end{abstract}

Key words. total irradiance - Sun - climate - global change - energy balance

\section{Introduction}

Total solar irradiance (TSI) is Earth's dominant energy input, exceeding the next largest energy source by nearly $10^{4}$ and exceeding all other sources combined by a factor of 2500 (Sellers 1965; Kren et al. 2014). Variations in solar irradiance produce natural forcing of Earth's climate with global- and regional-scale responses (IPCC 2013; Lean \& Rind 2008). Accurate and stable irradiance measurements are therefore critical for establishing the energy balance that determines Earth's climate (Trenberth et al. 2009; Loeb et al. 2012; Wild et al. 2013) and for reliable attribution of climate change, which requires discriminating natural from anthropogenic causes.

Total solar irradiance has been measured from space for an uninterrupted 35 years, providing the best record of the input radiative energy driving the Earth's climate system. Over a dozen instruments have contributed to this measurement record, as shown in Figure 1. This climate driver is observed to vary on scales of minutes and years and likely varies over much longer time periods; although definitive knowledge of such long-term variations, while critical for climate studies, is currently limited by instrument accuracy and stability. I describe the climatedriven measurement requirements and assess the spaceborne measurement record's accuracy and stability, including recent improvements to the record resulting from international collaboration calibration efforts.

\section{Irradiance measurement requirements for climate studies}

The 35 year TSI record clearly shows the effects of short-term solar activity such as the formation and disappearance of sunspots and faculae, and much of the observed short-term variability can be estimated by TSI proxy models based on these two components (see, for example, Fröhlich \& Lean 2004). More sophisticated irradiance models, such as those by
Fligge \& Solanki (2000), Krivova et al. (2003), Unruh et al. (1999), and Ball et al. (2011) include additional solar activity types to provide further refinements, but magnetic surface flux primarily from sunspots and faculae are the dominant drivers of TSI variability. As magnetic activity varies through the 11-year solar cycle, this periodicity shows prominently in TSI giving $\sim 0.08 \%$ increases during solar maximum (as evident in Fig. 1), and is of sufficient duration to influence climate (Gray et al. 2010; Lean 2010).

Being long-term by definition, climate would be influenced by solar changes over many years to centuries, and thus motivates a measurement record of greater duration and stability than the current spaceborne TSI record. Determining required measurement stabilities and accuracies relies on estimates of the magnitude of long-term solar variability as well as the Earth's climate sensitivity to solar variations. For instance, extremely high climate sensitivity to solar irradiance changes would require very precise monitoring of even small changes in the Sun's output; conversely, if solar variations over decadal scales were large and climate sensitivity to these changes relatively small compared to other influences, then solar measurement requirements could be less stringent.

Using multiple linear regression of several climate forcings, Lean (2010) reports climate sensitivities of $\sim 0.3 \mathrm{C} \mathrm{W}^{-1} \mathrm{~m}^{2}$ for solar forcing on decadal time scales, whereby a $0.1{ }^{\circ} \mathrm{C}$ global temperature change is caused by the $0.08 \%$ solar variations during a solar cycle. At times of its largest gradients, the solar cycle causes warming and cooling comparable to the rate of anthropogenic warming. (Fortunately, these solar variations are cyclic rather than monotonic.)

Less known is the solar variability on multi-decadal and century time scales. Estimates of such variability over the last 400 years, during which the sunspot measurement record provides a proxy for solar activity, are provided by Wang et al. (2005), Lean (2000), Tapping et al. (2007), Steinhilber et al. (2009), Solanki et al. (2013), Feulner (2011), and others, including a recent assessment by the IPCC AR5 Working 


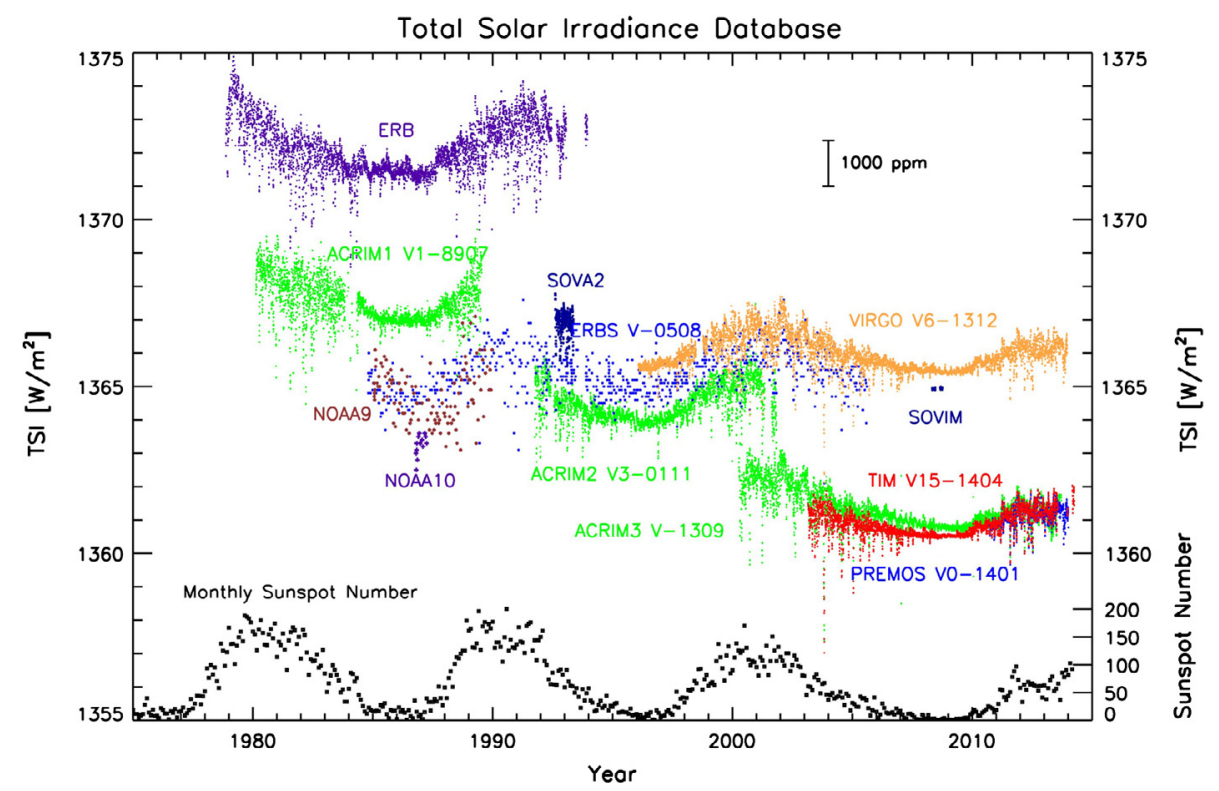

Fig. 1. Measurements of TSI from the instruments contributing to the current 35 year long spaceborne record are shown with each plotted on their reported scale after being normalized to 1 astronomical unit. Offsets are due to instrument calibration differences. The monthly sunspot numbers (black) show that the TSI varies in-phase with solar activity.

Group I (2013). Figure 2 shows the results of some of these models, all of which utilize solar proxies to extend the TSI record prior to the spacecraft measurement era.

The historical models of solar activity show the 11-year solar cycle in TSI as well as lower-frequency variations, the most prominent of which is the Maunder Minimum in the late $1600 \mathrm{~s}$. These long-term variations are more relevant for climate studies; but they are also somewhat tenuous, being based on proxies of TSI rather than actual measurements. Nevertheless, these models provide the best indications of potential long-term solar variations that must be measurable for discerning, via direct observations, current and future changes in the Sun's radiant output that can influence climate over decadal and longer time frames.

The models in Figure 2 suggest long-term solar variability rates of $0.05 \%-0.1 \%$ over nearly a century when entering or exiting the Maunder Minimum era. Direct detection of such changes in TSI thus requires instrument measurement stability less than $\sim 0.001 \%$ year $^{-1}$ and continual measurements. As discussed in Section 3.3, these stabilities are perhaps only achieved by the newest on-orbit TSI instruments, but not the older instruments contributing to the 35 year TSI spaceborne record. Alternatively, in the absence of the needed stability and measurement continuity, such solar trends can be detected via separate measurements over a long time period if sufficient instrument absolute accuracy is achieved. Detecting long-term variations such as entering or exiting the Maunder Minimum would in this case require accuracies of $\sim 0.01 \%$ and observations separated by decadal to multi-decadal times to allow sufficient TSI signal change to be detectable by disparate measurements of comparable accuracy.

These estimates of historical long-term solar variability thus drive the primary requirements for TSI measurements as needed for climate science, and are summarized in Table 1. Interestingly, two additional and independent means of deriving climate-driven TSI measurement requirements are described by Stevens et al. (2013) with very similar conclusions.

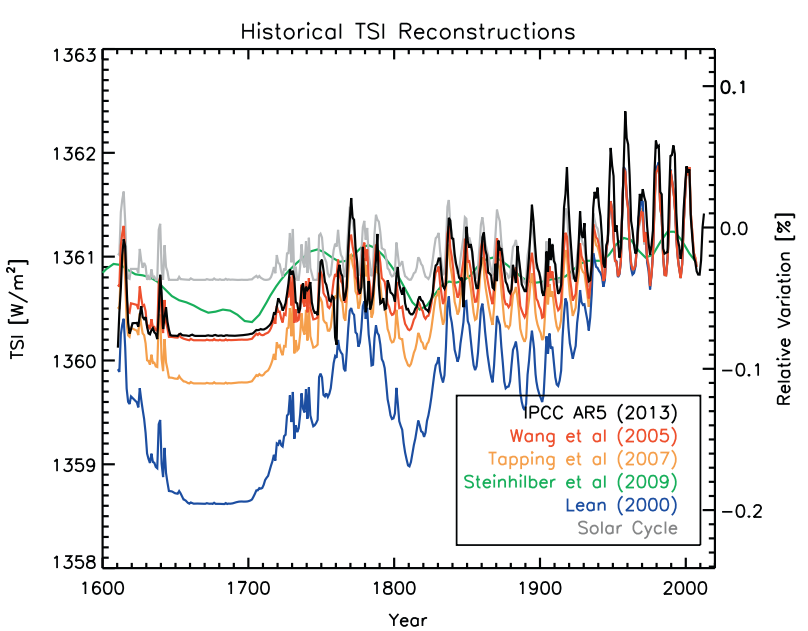

Fig. 2. Proxy models of solar variability over the last four centuries provide estimates of the change in the Sun's output over decades to centuries.

Table 1. TSI measurement requirements for climate studies.

\begin{tabular}{ll}
\hline \hline Measurement parameter & Requirement \\
\hline Absolute accuracy & $<0.01 \%$ \\
Stability & $<0.001 \%$ year $^{-1}$ \\
\hline
\end{tabular}

Daily measurements of spectral solar irradiance (SSI) across the visible and near-infrared began with NASA's SORCE mission (Rottman 2005). The spectral content of these irradiance measurements helps determine the solar activity types causing variability and the spectrally dependent effects on the Earth's atmosphere (Fontenla \& Harder 2005; Gray et al. 2010; Ball et al. 2011; Matthes 2011; and Ermolli et al. 2013, with a good review by Lean \& Woods 2010). Since both climate sensitivity and solar variability have spectral dependencies of which we are only beginning to learn via this new 
Table 2. TSI data sets evaluated.

\begin{tabular}{lcc}
\hline \hline Mission/instrument & Data version & Data date range \\
\hline ACRIMSat/ACRIM3 & daya2sddeg_ts4_Mar2013_hdr & 5 April 2000-4 March 2013 \\
PICARD/PREMOS & & 27 July 2010-31 March 2013 \\
SoHO/VIRGO & virgo_tsi_d_v6_002_1302 & 7 February 1996-1 January 2013 \\
SORCE/TIM & V.14 & 25 February 2003-30 July 2013 \\
\hline
\end{tabular}

record, the SSI measurement requirements are less well defined than for TSI. That is, since neither the long-term spectral solar variability nor the spectrally dependent Earth climate sensitivity are yet well known, the needed SSI accuracies and stabilities are less rigorously defined. However, since the visible and near-infrared spectral regions contribute most of the energy in the TSI that directly powers the Earth's climate system, given a lack of such direct solar variability and climate sensitivity knowledge from spectral irradiances presently, Table 1 might provide a reasonable initial estimate of the SSI measurement requirements for long-term climate studies. These levels of accuracy and stability are not achieved with the existing SSI instruments (Skupin et al. 2005; Harder et al. 2009), and there is considerable uncertainty in the long-term stabilities of the measurements, leading to disparate conclusions of solar variability even on solar cycle time scales (Matthes 2011; Lean $\&$ DeLand 2012). While perhaps not yet achieving the accuracy or stability requirements for true climate studies, the short duration visible and near-infrared SSI record is proving valuable for short-term solar variability effects on the Earth's atmosphere (Gray et al. 2010; Haigh 2011; Haigh et al. 2012).

Frequent spectral irradiance measurements at wavelengths shorter than $300 \mathrm{~nm}$ commenced with the Solar Mesosphere Explorer in 1981 followed by the Solar Backscatter Ultraviolet Instrument (SBUV/2) in 1984, the UARS/SOLSTICE and SUSIM instruments in 1991 (Rottman et al. 1993), and the SORCE/SOLSTICE in 2003 (McClintock et al. 2005a). Rottman (2005) summarizes the consensus from these measurements as: (1) the most energetic X-rays can vary by factors of 10 and larger, the EUV spectrum $(\lambda<120 \mathrm{~nm})$ by factors of 2 , and the UV spectrum $(\lambda<300 \mathrm{~nm})$ by up to $50 \%$; and (2) the spectrally integrated UV $(\lambda<300 \mathrm{~nm})$ variability accounts for roughly $30 \%$ of the solar cycle TSI variation with the remaining $70 \%$ attributed to the visible and infrared.

The relative solar variability at these shorter wavelengths is much greater than in the visible and the sensitivity of the Earth's atmosphere to variations in this spectral region is large. Therefore, based on the rationale given at the beginning of this section, climate-relevant measurement requirements at these shorter wavelengths are less stringent than given in Table 1. McClintock et al. (2005b) estimate needed absolute accuracies of $5 \%$ and stabilities of $0.5 \%$ year $^{-1}$ at wavelengths below $300 \mathrm{~nm}$.

Despite the more relaxed measurement requirements in the ultraviolet, the measurements themselves at these wavelengths remain difficult because of the lower overall signals and the increased susceptibility to instrument contamination resulting in on-orbit degradation and poor stability. As an example of the uncertainties in these measurements, the SORCE/SIM indicates much greater UV variability during the declining phase of Solar Cycle 23 than other instruments in the 250-300 nm range (Lean \& DeLand 2012), leading to disparate climate and atmospheric effects. Merkel et al. (2011) explore the results such discrepant measurements can have on atmospheric models compared to more traditional, proxy-based model solar inputs such as used by Marsh et al. (2007). As in the visible and near-infrared, improvements to ultraviolet SSI measurement accuracies and stabilities as well as a continued measurement record will improve uncertainties at these shorter wavelengths and our resultant understanding of their climate effects.

\section{Assessment of current TSI record}

Spaceborne TSI measurements, shown in Figure 1, began in 1978 and have continued uninterrupted since. Measurement overlap helps correct data offsets due to calibration differences between instruments. The primary causes of the offsets among the different TSI instruments are uncorrected internal scatter, which causes erroneously high readings, and incorrect optical power measurement (Kopp \& Lean 2011). Since the needed levels of absolute accuracy (Table 1) have not yet been demonstrated on orbit, this 35 year solar climate data record currently relies on continuity from stable instruments; although we will see that the needed stabilities are not necessarily proven on orbit either.

The spaceborne TSI record's most recent contributions are from ESA's SoHO/VIRGO ${ }^{1}$, NASA's ACRIMSat/ACRIM3 ${ }^{2}$, NASA's SORCE/TIM ${ }^{3}$, and CNES's PICARD/PREMOS ${ }^{4}$. SoHO was launched in late 1995, ACRIMSat in late 1999, SORCE in early 2003, and PICARD mid-year in 2010. All have provided nearly daily data. (PICARD/SOVAP ${ }^{5}$ data are not publicly available, so this instrument has not contributed to the research community's TSI record.) The latest data versions and the date ranges used for evaluation of each instrument are provided in Table 2 and plotted in Figure 3. These data of daily TSI values were provided by each instrument team's routine data processing system.

\subsection{Instrument accuracy}

Kopp \& Lean (2011) summarize the large amount of progress over the last few years to understand the offsets (the differences on an absolute scale) between the different TSI instruments. Several large offsets in prior data compilations have now been corrected, giving the good agreement seen in Figure 1 between the TIM, ACRIM3, and PREMOS. Most of the improved understanding is due to international collaborations using the

\footnotetext{
${ }^{1}$ European Space Agency's Solar and Heliospheric Observatory/ Variability of Solar Irradiance and Gravity Oscillations.

2 National Aeronautics and Space Administration's ACRIMSat/ Active Cavity Radiometer Irradiance Monitor.

${ }^{3}$ NASA's Solar Radiation and Climate Experiment/Total Irradiance Monitor.

${ }^{4}$ Centre National d'Etudes Spatiales (French Space Agency's) Picard/PREcision MOnitor Sensor.

${ }^{5}$ Centre National d'Etudes Spatiales (French Space Agency's) Picard/SOlar VAriability Picard.
} 


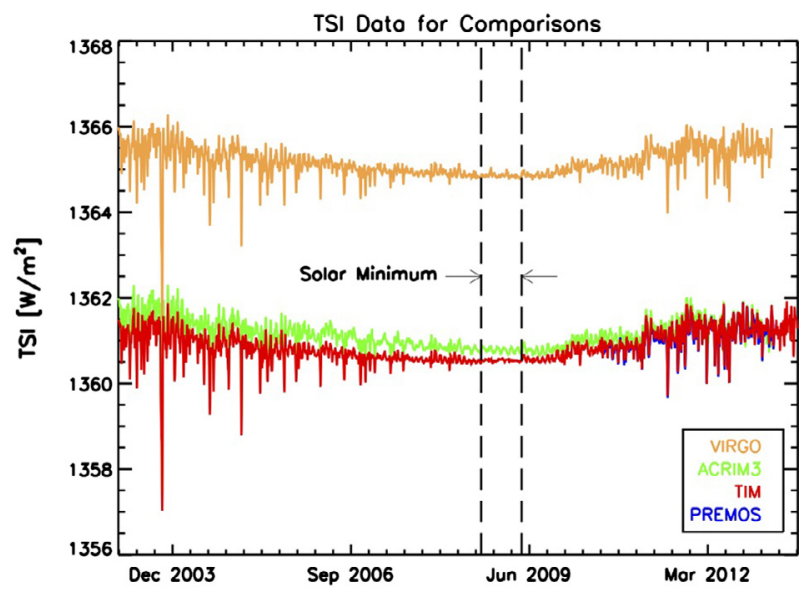

Fig. 3. Time series of TSI data from ACRIM3, PREMOS, TIM, and VIRGO since 2003. These are the instrument data used in this paper's analyses.

new TSI Radiometer Facility (TRF), a cryogenic radiometer based ground calibration facility able to characterize TSI instruments under flight-like conditions of full solar irradiance levels while operating in vacuum (Kopp et al. 2007).

A large fraction of the offsets causing higher readings in older versions of ACRIM3 data and current VIRGO data is due to internal optical scatter within these two instruments, which have a different aperture and baffle layout than that of the TIM (layout described by Kopp \& Lawrence 2005) and allow two to three times more sunlight to enter the instrument than is ultimately measured. The PREMOS has the same issue with high scatter, but this effect was characterized prior to launch for that instrument (Fehlmann et al. 2012). The newest instruments, the TIM and the PREMOS, have stated relative uncertainties of $0.035 \%$ and $0.03 \%$ respectively. These uncertainties are lower than any prior instrument but still fail to meet the accuracy requirement in Table 1 . Thus the climate data record continues to rely on instrument stability and continuity.

\subsection{Instrument noise}

Overall the data from the four instruments show similar trends. Each tracks the solar cycle and each has similar responses to short-term solar variability due to sunspots or faculae. The 2008 solar minimum, a time when the Sun was fairly quiet, provides a good opportunity to gain insights into instrument artifacts. By comparing data from the three instruments observing during this minimum, when they were simultaneously observing the same, relatively quiet source, signals that are not common to all three indicate likely instrument artifacts. This solar minimum time period is expanded in Figure 4.

The signals plotted in Figure 4 include both the noise from each instrument and actual solar variations. Common mode variations between instruments likely indicate true solar variations, whereas differences are instrument artifacts. Two prominent such artifacts are: two positive spikes in the VIRGO data, which coincide with SoHO spacecraft Keyhole pointing maneuvers; and a large oscillation in the ACRIM3 data (see also Figure 11), the cause of which is currently unknown but may be improperly corrected instrument thermal variations.

The standard deviations of each instrument's daily TSI values during solar minimum, being the quadrature sum of

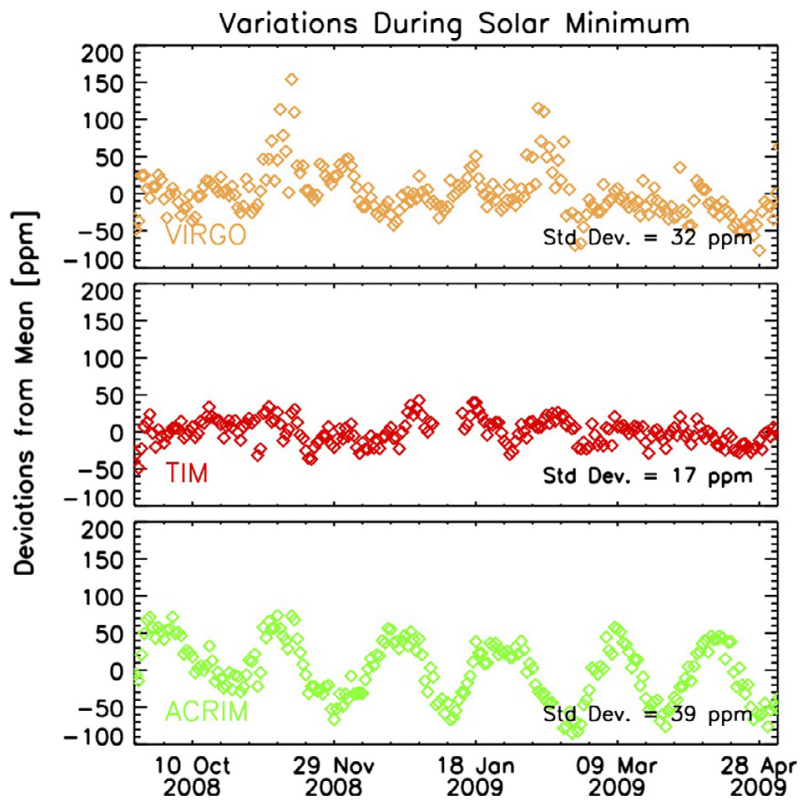

Fig. 4. The reported TSI values from the VIRGO (top), TIM (middle), and ACRIM3 (bottom) are plotted relative to their means during the 20 Sept. 2008 to 5 May 2009 time period of very little solar activity during the 2008 solar minimum. Differences between the three instruments indicate instrument artifacts, as the Sun was very quiet during this period.

the instrument noise and the actual solar variability, are listed with each plot in Figure 4. These are given in parts per million (ppm, or one part in $10^{6}$ ) of the total $1361 \mathrm{~W} \mathrm{~m}^{-2}$ TSI signal. The TIM's noise is lower than that of any other flight instrument partially because it is the only instrument utilizing phase sensitive detection in either on-orbit thermal control or ground data processing. Independent calibration measurements give $<4 \mathrm{ppm}$ as the TIM instrument noise (Kopp et al. 2005), suggesting that actual solar activity during this solar minimum time range accounts for nearly all of the $17 \mathrm{ppm}$ variability indicated in the figure. The larger variations in the ACRIM3 and VIRGO data during this time period imply that their solar minimum measurements are dominated by instrument noise rather than solar variability. Estimates of the noise range of each instrument's daily data are given in Table 3, with the range allowing for up to $17 \mathrm{ppm}$ of the variability for each instrument's data in Figure 4 to be due to solar variability.

The PREMOS data, shown in Figure 5, did not begin until mid-2010 and thus cannot be compared during solar minimum. This instrument agrees within uncertainties with the SORCE/ TIM on an absolute scale. The two instruments have small long-term relative variations, indicating similar levels of measurement stability. The standard deviation of the differences between the two (shown in the lower plot in Fig. 5) is the quadrature sum of the TIM and the PREMOS noise levels, from which the PREMOS noise level range can be estimated (see Table 3). This level of agreement is pleasing to see, particularly since these are two very different instrument designs, with the PREMOS being a 1990s era VIRGO-like design and the TIM having the newer low-scatter optical arrangement explained by Kopp \& Lean (2011), indicating that quality TSI measurements are achievable with either design given good ground calibrations. 
Table 3. Instrument performance.

\begin{tabular}{lccc}
\hline \hline Mission/Instrument & Stated accuracy $(\mathrm{ppm})$ & Stability* $\left.^{*} \mathrm{ppm}_{\text {year }}^{-1}\right)$ & Noise* $^{*}\left(\mathrm{ppm}^{-}\right)$ \\
\hline ACRIMSat/ACRIM3 & 1000 & 61 & $36-39$ \\
PICARD/PREMOS & 300 & 34 & $28-33$ \\
SoHO/VIRGO & 1000 & 27 & $28-32$ \\
SORCE/TIM & 350 & 10 (est.) & $4-17$ \\
\hline
\end{tabular}

* Based on analyses presented in this study (rather than the instruments' stated values).
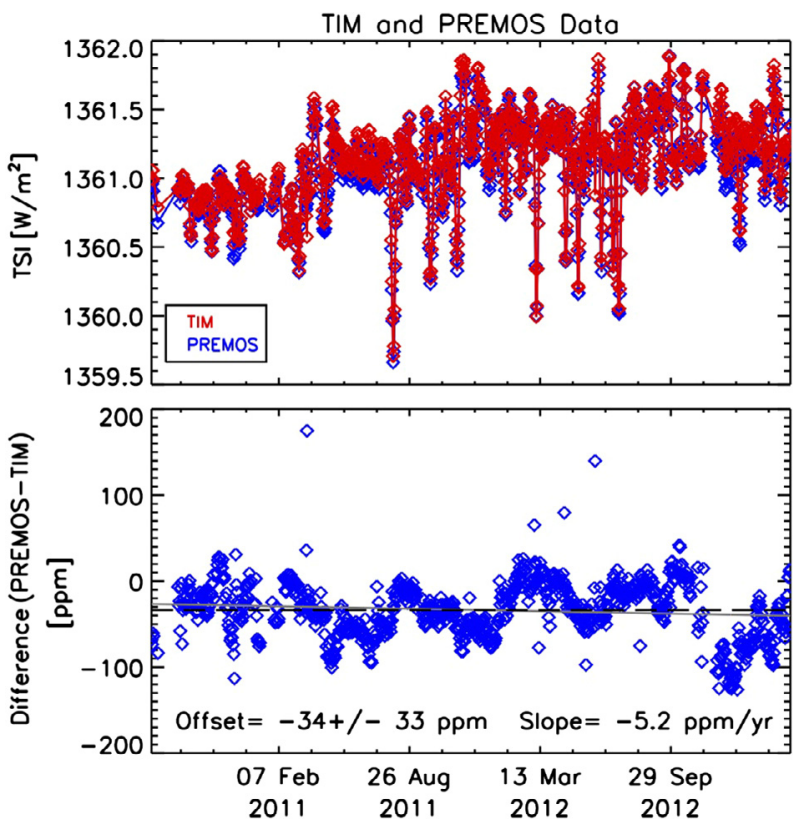

Fig. 5. The TSI values from the PREMOS (blue) and TIM (red) instruments are shown in the upper plot with differences between the two in the lower plot. The two instruments agree very well in both absolute value and stability over the temporal extent of the PREMOS data, which started mid-year in 2010 .

\subsection{Instrument stability}

Climate studies rely on long-term TSI records which are created by combining the overlapping measurements of individual shorter-duration instrument records to create a single time series composite. Differing trends reported by different instruments can have a significant effect on this composite. Hence, in climate studies estimating sensitivity to solar variability, the choice of TSI instrument for different time ranges used in creating a composite has a large effect on the solar trends in that composite.

The two most prominent TSI composites are those of Fröhlich (2000 with updates from ftp://ftp.pmodwrc.ch/pub/ data/irradiance/virgo/TSI/) and Willson (http://www.acrim. com/Data\%20Products.htm), and are shown in Figure 6. These show greatly varying trends over a solar cycle, as is most evident by the relative differences between the solar minima values in 1985 and 1996 in each composite. Choice of composite can thus lead to very different estimated contributions of solar forcing to increasing global temperatures during that time range.

An International Space Studies Institute (ISSI) team has been formed to bring together the primary personnel representing each instrument as well as TSI data users and modelers to try to understand and correct for instrument artifacts. The goal of this team is to create a new composite with consensus from

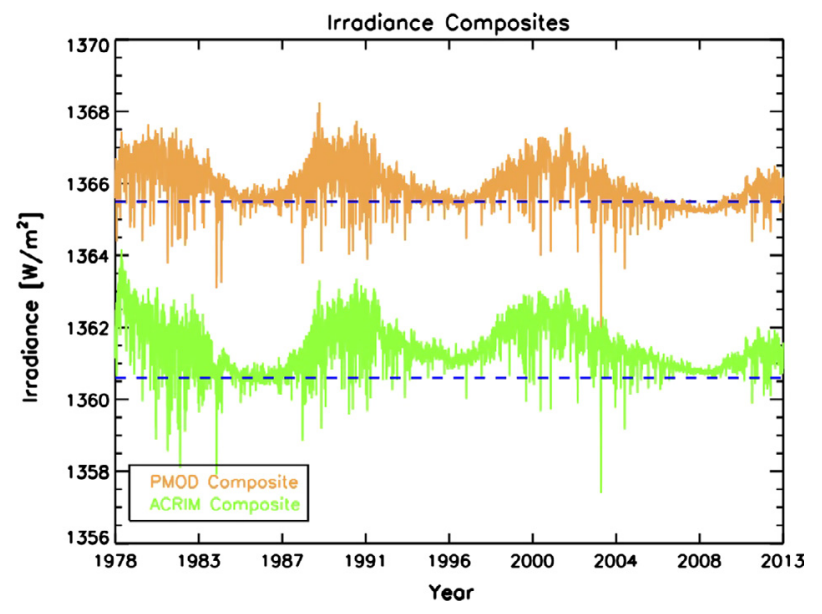

Fig. 6. The TSI composites created by Fröhlich (orange) and Willson (green) give different trends between solar minima because of differing long-term trends in the instruments used to create each. The horizontal dashed lines highlight these minimum-to-minimum differences.

the active TSI community rather than the disparate composites currently created by individual teams. One important addition this new composite will have that is lacking in the current composites is a time-dependent uncertainty associated with the daily composite TSI values, with this uncertainty reflective of the quality of data from different temporal periods in the spacecraft records. Such an uncertainty will allow composite data users to readily identify times where the data record is better or more poorly known.

Each of the four instruments currently contributing to the TSI record monitors on-orbit degradation by duty cycling redundant radiometers. This method, initiated with the ACRIM1 in 1980 (Willson 1979), enables tracking of degradation due to solar exposure of the primary radiometer via intermittent inter-comparisons with lesser-used secondary or even tertiary radiometers, with subsequent correcting in ground processing. The degradations of the ACRIM3, PREMOS, TIM, and VIRGO are shown in Figure 7 through Figure 10 respectively.

These tracked instrument degradation measurements are corrected in released data products, providing measurement stability to the extent that they truly represent all instrument sensitivity changes. Stability uncertainty depends on the accuracy to which these changes are known. To some extent, the uncertainty in the degradation correction might be expected to increase with the magnitude of the total correction; for example, an inherently unchanging instrument would have no needed correction and hence no associated uncertainty associated with that correction. 
ACRIMSAT/ACRIM3 Degradation Calibration

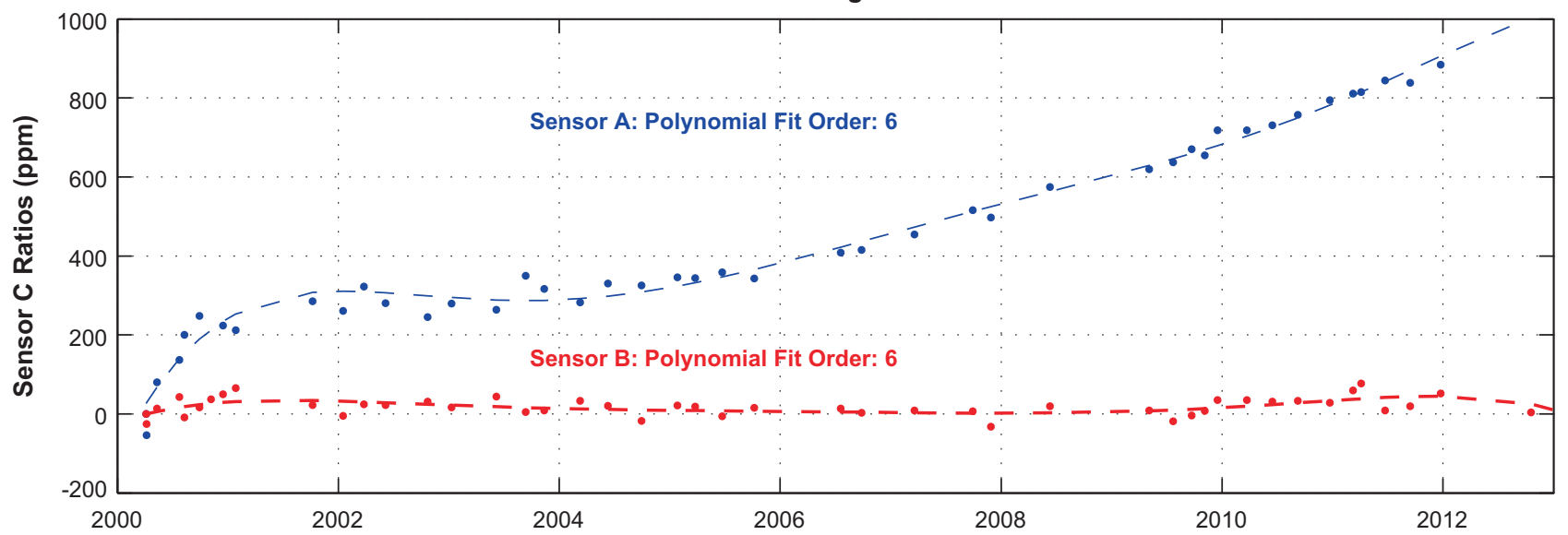

Fig. 7. Changes in ratios of simultaneous TSI measurements with the ACRIM3's Sensor A (primary radiometer) or Sensor B (secondary radiometer) to the least used Sensor C allow tracking of changes due to solar exposure in the two more frequently used sensors. Sensor A has degraded by $1000 \mathrm{ppm}$ over the 13 years on orbit. This degradation is corrected using a 6th order polynomial fit in released TSI values from the instrument (plot from acrim.com).

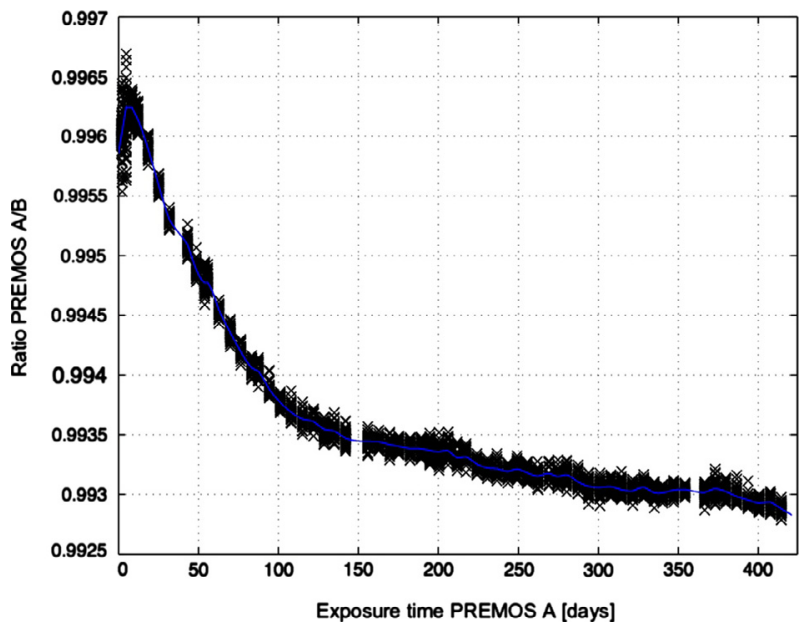

Fig. 8. Ratios of TSI measurements with the PREMOS's Sensor A (primary radiometer) to its Sensor B (backup radiometer) track solar exposure dependent changes in the primary, which has degraded by $3500 \mathrm{ppm}$ after an initial $400 \mathrm{ppm}$ increase at the beginning of the mission. This is a greater change than any other instrument experienced in the first two years of operation (plot courtesy of PREMOS team).

Instrument stability is also affected by other factors than those monitored by comparisons of redundant radiometers, a method which only detects changes indicated by differences between radiometers, such as solar exposure dependent degradation of a primary radiometer. Common mode changes to electronics, aperture size or thermal properties, instrument thermal emissivities, and surface scattering properties are examples of effects that may not be detected by intra-instrument comparisons between redundant radiometers.

Inter-instrument comparisons provide insight into relative instrument stabilities, since the spaceborne instruments view the Sun at similar times and should thus measure similar TSI values and trends. The lower plot in Figure 5 shows the difference, or offset, between the TIM and the PREMOS time series. These two, the newest of the spaceborne TSI instruments, agree on the absolute value of TSI to well within the quoted uncertainties $(\sim 300 \mathrm{ppm})$ of both instruments. Similarly the

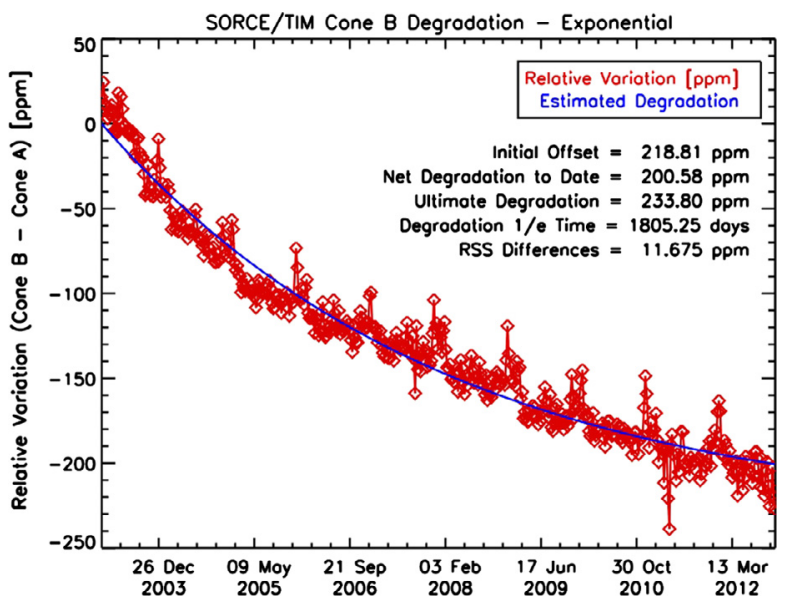

Fig. 9. Relative changes in ratios of simultaneous TSI measurements with the TIM's Cone B (primary radiometer) to its Cone A (secondary radiometer) indicate solar exposure dependent changes in the primary. The TIM shows lower degradation than any other flight radiometer, with only $200 \mathrm{ppm}$ of change in the primary cavity after 10 years on orbit. This degradation very nearly follows a classic exponential, with a fit shown (blue).

long-term trends between the two agree to within nearly 5 ppm year ${ }^{-1}$, indicating similar levels of stability.

Figure 11 similarly shows differences between the TIM and the VIRGO (upper plot) and the ACRIM3 (lower plot). The long-term differences between the instruments indicate that both the VIRGO's and ACRIM3's TSI values are decreasing with time faster than observed by the TIM (and thus also the PREMOS). These trends are 16 and $33 \mathrm{ppm} \mathrm{year}^{-1}$ for VIRGO and ACRIM3 respectively relative to the TIM, so not all instruments are achieving the desired $10 \mathrm{ppm}_{\text {year }}{ }^{-1}$ stability level. The standard deviation of the slope differences between all four instruments is $14.6 \mathrm{ppm}_{\text {year }}{ }^{-1}$, meaning that the collective of instruments does not achieve the required stability level for climate studies given in Table 1 .

Relative comparisons such as those in Figure 5 and Figure 11 unfortunately cannot indicate which instrument is the more stable over long time frames. Analysis over shorter 


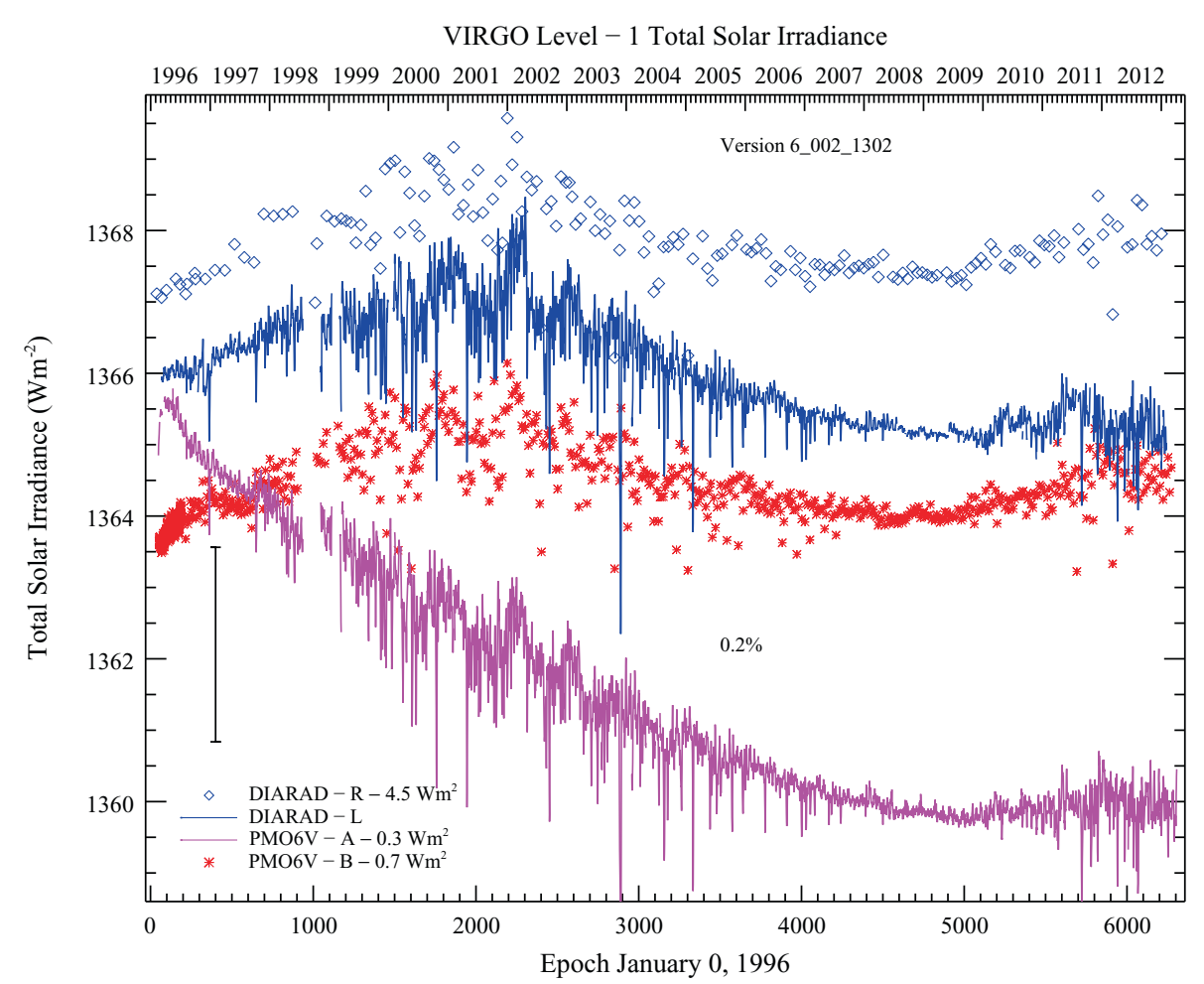

Fig. 10. The VIRGO's primary channel's TSI values (PMO6V-A, shifted downward by $0.3 \mathrm{~W} \mathrm{~m}^{-2}$ ) are shown in magenta. This channel's sensitivity has decreased by more than $4000 \mathrm{ppm}$ following an initial $500 \mathrm{ppm}$ increase at the beginning of the mission. This degradation pattern is very similar to that of the PREMOS, although the VIRGO's degradation occurred over a much longer time range. (The blue line shows the VIRGO's DIARAD-R channel's irradiance measurements shifted downward by $4.5 \mathrm{~W} \mathrm{~m}^{-2}$, the blue diamonds indicate the lesser-used DIARAD-L, and the red asterisks the lesser-used PMO6V-B shifted downward by $1.7 \mathrm{~W} \mathrm{~m}^{-2}$.) (plot courtesy of VIRGO team)

time scales however can indicate artifacts in individual instruments, which may lend some insight into which are the more stable on longer time scales. In addition to the long-term differences, Figure 11 shows short-term differences between the three instruments. Such short-term variations that are not common mode to the three are likely artifacts isolated to an individual instrument. The 90-day spikes seen in the VIRGO data (but not in the TIM or ACRIM3 data) and the varying periodicity oscillations in the ACRIM3 data (but not in the TIM's or VIRGO's) mentioned in Section 3.1 are yet more apparent in the longer duration plots of Figure 11.

There is no independent on-orbit reference against which to compare each instrument for stability, so only inter-instrument comparisons can be made. Since the TIM has the best inherent stability of the four instruments (i.e. the lowest degradation, per Figure 9) and lacks obvious short duration artifacts such as those of the VIRGO and ACRIM3 in Figure 11, I select it as the relative reference for these inter-instrument comparisons. Its stability uncertainty is estimated at $10 \mathrm{ppm}_{\text {year }}{ }^{-1}$ (Kopp et al. 2005). Using the TIM as a reference for shorter-duration comparisons with other instruments shows that even aside from the long duration slope differences of Figure 11, there remain unexplained variations that are not common mode between all instruments, suggesting again that the desired stability levels are not uniformly achieved.

Figure 12 shows a 1-year smoothing of the residuals from Figure 5 and Figure 11. Differences between the instruments, even smoothed, are not common mode, again suggesting that instrument artifacts are limiting measurement stabilities. The variations in slope of these smoothed residuals provides

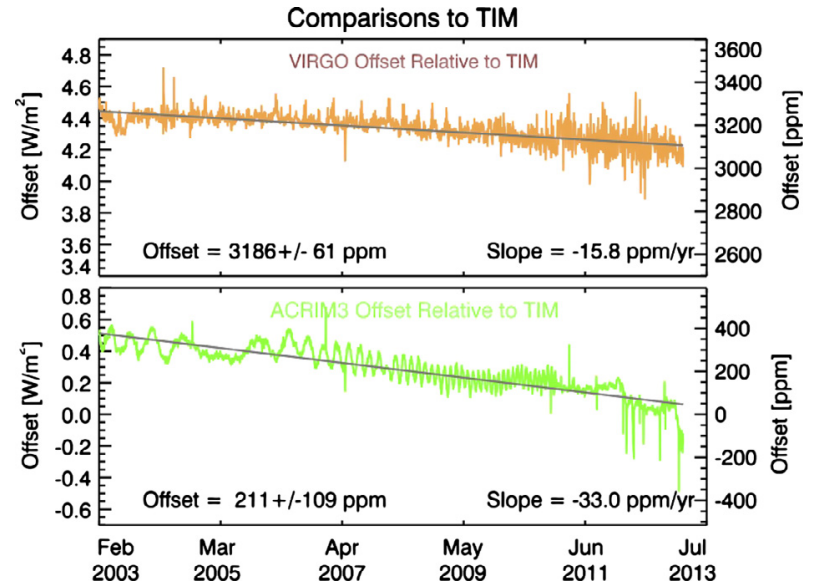

Fig. 11. TSI differences between the VIRGO (upper plot) and the ACRIM3 (lower plot) relative to the TIM indicate long-term stability differences between the three instruments.

a quantitative indicator of the stability uncertainties between instruments. These values are given in Figure 12 and summarized in Table 3.

\section{Future TSI Measurements}

All four spaceborne instruments in Table 3 have exceeded their intended mission durations. The SoHO mission is approaching 18 years in space and communications with the 14-year old 

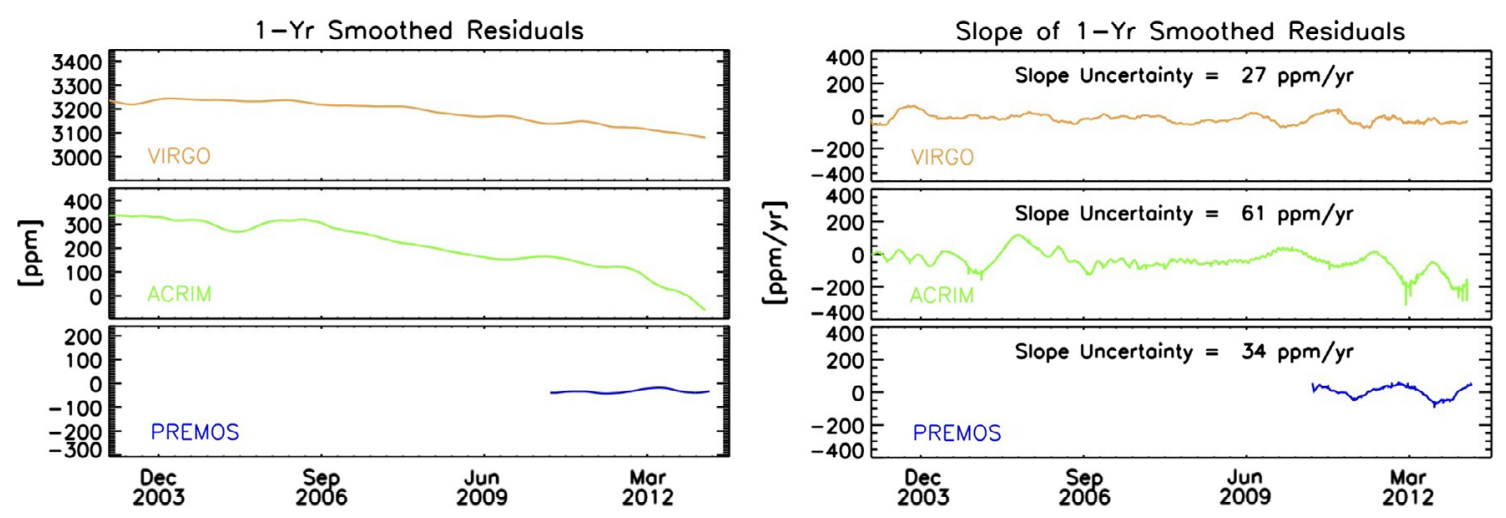

Fig. 12. One-year smoothings of the differences in Figure 5 and Figure 11 are shown in the left-hand plot, highlighting gradual instrument variations with respect to the TIM. The slope of each difference is shown in the right-hand plot. Relative differences between the instruments that are not common mode indicate instrument artifacts. The standard deviations of the slopes are indicators of instrument stability uncertainty.

ACRIMSat ceased in Nov. 2013. SORCE has doubled its 5 -year lifetime goal but battery issues have limited recent regular daily solar measurements. The newest mission, PICARD, was intended for a shorter duration and was decommissioned in early 2014. NASA's Glory mission, built to continue the measurement record with an improved TIM instrument from that on SORCE, was lost due to a vehicle failure at launch. The JPSS (formerly NPOESS) program flying the TSIS/TIM has had repeated schedule delays and is not due for launch prior to mid-2017. Since the data record currently relies on measurement stability and continuity, the possibility of a gap of high-quality stable TSI measurements is appearing quite possible and would limit the value of this solar record for climate studies. Fortunately, two upcoming shortduration missions may help.

The JPSS TSI Continuity Transfer Experiment (TCTE) launched a TIM instrument in Nov. 2013 as part of the Air Force mission STP-Sat3. Built alongside the original SORCE/ TIM but with newer and better calibrations than possible at the time of the SORCE launch, such as those on the TRF, this instrument should improve the measurement record's absolute accuracy - particularly via direct overlap obtained with the SORCE/TIM - while providing similar stability levels to that instrument (Kopp \& Lean 2013; Privette et al. 2013). One orbit of solar measurements per day are planned (rather than the near full-time measurements of the existing instruments on more solar-dedicated missions).

The Norwegian Space Center's NORSAT-1 will be flying a version of the PMOD's Digital Absolute RAdiometer (DARA) instrument after further development to reduce that groundbased instrument's power, mass, and volume. This Compact Lightweight Absolute Radiometer (CLARA) is intended for launch in 2015 .

Both the TCTE and the CLARA are opportunistic missions to extend the TSI measurement record. JPSS is the only program worldwide planning a long-term continued means of acquiring TSI data, but this program has allowed schedule slips that risk record continuity. As climate is an international concern and these solar measurements are critical for discerning natural from anthropogenic climate forcings, a more cohesive and scheduledriven program is needed, preferably at an international level.

\section{Summary}

The TSI record provides the most radiometrically accurate and stable measurements of net solar inputs needed for Earth climate studies from its uninterrupted spaceborne measurements over the last 35 years. Further instrument and calibration improvements affecting both accuracy and stability should help meet the demanding climate-driven measurement requirements of the record and enable improved climate studies via better estimates of long-term solar variability. In the short term, two imminent missions will be continuing the TSI measurement record, but better and more cohesive national and/or international long-term programs are needed to avoid possible gaps in this critical solar climate data record.

Acknowledgements. I greatly appreciate the thoroughness of this publication's reviewers, particularly Dr. W. Ball of Imperial College and an anonymous reviewer, for their thoughtful suggestions and the resulting clarity improvements. This work was supported by NOAA's "Developing a Climate Data Record for Total and Spectral Solar Irradiance" (NA09NES4400016) and NASA's SORCE (NAS5-97045) projects.

\section{References}

Ball, W.T., Y.C. Unruh, N.A. Krivova, S. Solanki, and J.W. Harder, Solar irradiance variability: a six-year comparison between SORCE observations and the SATIRE model, $A \& A, \mathbf{5 3 0}, \mathrm{A} 71$, DOI: 10.1051/0004-6361/201016189, 2011.

Ermolli, I., K. Matthes, T. Dudok de Wit, N.A. Krivova, K. Tourpali, et al., Recent variability of the solar spectral irradiance and its impact on climate modelling, Atmos. Chem. Phys., 13, 3945-3977, DOI: 10.5194/acp-13-3945-2013, 2013.

Fehlmann, A., G. Kopp, W. Schmutz, R. Winkler, W. Finsterle, and N. Fox, Fourth world radiometric reference to SI radiometric scale comparison and implications to On-orbit measurements of the total solar irradiance, Metrologia, S34-S38, DOI: 10.1088/0026-1394/49/2/S34, 2012.

Feulner, G., Are the most recent estimates for Maunder Minimum solar irradiance in agreement with temperature reconstructions?, Geophys. Res. Lett., 38, L16706, DOI: 10.1029/2011GL048529, 2011.

Fligge, M., and S.K. Solanki, Properties of flux tubes and the relation with solar irradiance variability, J. Astrophys. Astron., 21, 275-282, DOI: 10.1007/BF02702409, 2000.

Fontenla, J., and G. Harder, Physical modeling of spectral irradiance variations, Memorie della Societa Astronomica Italiana, 76, 826-833, 2005.

Fröhlich, C., Observations of irradiance variations, Space Sci. Rev., 94, 15-24, DOI: 10.1023/A:1026765712084, 2000.

Fröhlich, C., and J. Lean, Solar radiative output and its variability: evidence and mechanisms, Astron. Astrophys. Rev., 12, 273-320, DOI: 10.1007/s00159-004-0024-1, 2004. 
Gray, L.J., J. Beer, M. Geller, J.D. Haigh, M. Lockwood, et al., Solar influences on climate, Rev. Geophys., 48, RG4001, DOI: 10.1029/2009RG000282, 2010.

Haigh, J.D., A.R. Winning, R. Toumi, and J.W. Harder, An influence of solar spectral variations on radiative forcing of climate, Nature, 467, 696-699, DOI: 10.1038/nature09426, Oct. 2012.

Haigh, J., Solar influences on climate, Grantham Institute for Climate Change, Briefing Paper No. 5, February 2011.

Harder, J.W., J.M. Fontenla, P. Pilewskie, E.C. Richard, and T.N. Woods, Trends in solar spectral irradiance variability in the visible and infrared, Geophys. Res. Lett., 36, L07801, 1-5, DOI: 10.1029/2008GL036797, 2009.

IPCC, Climate change 2013: the physical science basis, Contribution of Working Group I to the Fifth Assessment Report of the Intergovernmental Panel on Climate Change, Cambridge University Press, 2013.

Kopp, G., and G. Lawrence, The total irradiance monitor (TIM): instrument design, Sol. Phys., 230 DOI: $10.1007 / \mathrm{s} 11207-005-7446-4,2005$.

Kopp, G., K. Heuerman, and G. Lawrence, The total irradiance monitor (TIM): instrument calibration, Sol. Phys., 230, 111-127, DOI: 10.1007/s11207-005-7447-3, 2005.

Kopp, G., K. Heuerman, D. Harber, and V. Drake, The TSI radiometer facility - absolute calibrations for total solar irradiance instruments, SPIE Proceedings, 6677-09, DOI: 10.1117/12.734553, 2007.

Kopp, G., and J.L. Lean, A new, lower value of total solar irradiance: evidence and climate significance, Geophys. Res. Lett., 38, L01706, DOI: 10.1029/2010GL045777, 2011.

Kopp, G., and J. Lean, The solar climate data record: scientific assessment of strategies to mitigate an impending gap in total solar irradiance observations between the NASA SORCE and NOAA TSIS missions (Study B), NRC Report solicited by NOAA NCDC, January 2013.

Kren, A.C., P. Pilewskie, and O. Coddington, An examination of energy sources for Earth's Atmosphere, Rev. Geophys., 2014 (in preparation).

Krivova, N.A., S.K. Solanki, M. Fligge, and Y. C. Unruh, Reconstruction of solar irradiance variations in cycle 23: Is solar surface magnetism the cause?, $A \& A, 399, \mathrm{~L} 1-\mathrm{L} 4$, DOI: 10.1051/0004-6361:20030029, 2003.

Lean, J., Evolution of the sun's spectral irradiance since the maunder minimum, Geophys. Res. Lett., 27 (16), 2425-2428, DOI: 10.1029/2000GL000043, 2000.

Lean, J. L., and D. H. Rind, How natural and anthropogenic influences alter global and regional surface temperatures: 1889 to 2006, Geophys. Res. Lett., 35, L18701, DOI: $10.1029 / 2008$ GL034864, 2008.

Lean, J.L., and T.N. Woods, Solar total and spectral irradiance measurements and models: a users guide. in Evolving solar physics and the climates of earth and space, K., Schrijver, and G. Siscoe (Eds.) Cambridge Univ. Press, 2010.

Lean, J., Cycles and trends in solar irradiance and climate, WIRES Climate Change, 1, 111-122, DOI: 10.1002/wcc.018, 2010.

Lean, J.L., and M.T. DeLand, How does the sun's spectrum vary?, Journal of Climate, 25, 2555-2560, DOI: 10.1175/JCLI-D-11-00571.1, 2012.

Loeb, N.G., J.M. Lyman, G.C. Johnson, R.P. Allan, D.R. Doelling, T. Wong, B.J. Soden, and G.L. Stephens, Observed changes in top-of-the-atmosphere radiation and upper-ocean heating consistent with uncertainty, Nat. Geosci., 1, 110-113, DOI: 10. 1038/ngeo1375, 2012.

Marsh, D.R., R.R. Garcia, D.E. Kinnison, B.A. Boville, F. Sassi, S.C. Solomon, and K. Matthes, Modeling the whole atmosphere response to solar cycle changes in radiative and geomagnetic forcing, J. Geophys. Res., 112, D23306,

DOI: 10.1029/2006JD008306, 2007.

Matthes, K., Solar cycle and climate predictions, Nat. Geosci., 735-736, News and Views, DOI: 10.1038/ngeo1298, 2011.

McClintock, W.E., M. Snow, and T.N. Woods, Solar-Stellar Irradiance Comparison Experiment II (SOLSTICE II): instrument concept and design, Sol. Phys., 230, 225-258, DOI: 10.1007/s11207-005-7446-4, 2005.

McClintock, W.E., M. Snow, and T.N. Woods, Solar-Stellar Irradiance Comparison Experiment II (SOLSTICE II): pre-launch and on-orbit calibrations, Sol. Phys., 230, 259-294, DOI: 10.1007/s11207-005-7446-4, 2005.

Merkel, A.W., J.W. Harder, D.R. Marsh, A.K. Smith, J.M. Fontenla, and T.N. Woods, The impact of solar spectral irradiance variability on middle atmospheric ozone, Geophys. Res. Lett., 38, L13802, DOI: 10.1029/2011GL047561, 2011.

Privette, J., S. Walters, G. Kopp, J. Lean, and R. Cahalan, Mitigating a likely gap in total solar irradiance measurements between the SORCE and TSIS missions, in: AMS Meeting, Austin, TX, 6-10 January 2013.

Rottman, G.J., T.N. Woods, and T.P. Sparn, Solar Stellar Irradiance Comparison Experiment I: 1 instrument design and operation, J. Geophys. Res., 98, 10667-10677, DOI: 10.1029/93JD00462, 1993.

Rottman, G., The SORCE Mission, Sol. Phys., 203 (1), 7-25, DOI: $10.1007 / \mathrm{s} 11207-005-8112-6,2005$.

Sellers, W.D., Physical Climatology, Univ. of Chicago Press, p. 12, 1965.

Skupin, J., M. Weber, S. Noel, H. Bovensmann, and J. P. Burrows, GOME and SCIAMACHY solar measurements: solar spectral irradiance and $\mathrm{Mg}$ II solar activity proxy indicator, Memorie della Societa Astronomica Italiana, 76, 1038, 2005.

Solanki, S.K., N.A. Krivova, and J.D. Haigh, Solar irradiance variability and climate, Annual Review of Astronomy and Astrophysics, 51, 311-351,

DOI: 10.1146/annurev-astro-082812-141007, 2013.

Steinhilber, F., J. Beer, and C. Fröhlich, Total solar irradiance during the Holocene, Geophys. Res. Lett., 36, L19704, DOI: 10.1029/2009GL040142, 2009.

Stevens, G., J.D. Haigh, J.W. Harvey, C. Ichoku, K.-N. Liou, J. Rice, W. Smith, and B. Wielicki, Review of NOAA Working Group Report on Maintaining the Continuation of Long-term Satellite Total Solar Irradiance Observation, National Academies Press, 978-0-309-28763-0, 2013

Tapping, K.F., D. Boteler, P. Charbonneau, A. Crouch, A. Manson, and $\mathrm{H}$. Paquette, Solar magnetic activity and total irradiance since the maunder minimum, Sol. Phys., 246, 309-326, DOI: 10.1007/s11207-007-9047, 2007.

Trenberth, K., J.T. Fasullo, and J. Kiehl, Earth's global energy Budget, Bull. Am. Meteorol. Soc., 311-323, DOI: 10.1175/2008BAMS2634.1, 2009.

Unruh, Y.C., S.K. Solanki, and M. Fligge, The spectral dependence of facular contrast and solar irradiance variations, $A \& A, \mathbf{3 4 5}$, 635-642, 1999.

Wang, Y.-M., J. L. Lean, and N. R. Sheeley Jr., Modeling the Sun's Magnetic Field and Irradiance Since 1713, Astrophys. J., 625, 522-538, DOI: 10.1086/429689, 2005.

Wild, M., D. Folini, C. Schär, N. Loeb, E.G. Dutton, and G. KönigLanglo, A new diagram of the global energy balance, American Institute of Physics Conference Proceedings, 1531, 628-631, DOI: 10.1063/1.4804848, 2013.

Willson, R.C., Active cavity radiometer type IV, Appl. Opt., 18 (2), 179-188, DOI: 10.1364/AO.18.000179, 1979.

Cite this article as: Kopp G: An assessment of the solar irradiance record for climate studies. J. Space Weather Space Clim., 2014, 4, A14. 